\section{PRESUPUESTO PARTICIPATIVO, ¿LAS REGIONES MÁS VULNERABLES LO INVIERTEN EN SALUD?}

\author{
PARTICIPATIVE BUDGET: DO THE MOST \\ VULNERABLE REGIONS INVEST \\ IT IN HEALTH CARE?
}

\section{Rodrigo M. Carrillo-Larco ${ }^{1,2, a}$, Eddy R. Segura, ${ }^{3, b}$}

Los países latinoamericanos han adoptado un modelo de gestión en el que la población coordina con sus autoridades los planes de inversión social (1); a ello se le conoce como presupuesto participativo (PP). En el Perú, la Ley 28056, junto con su reglamento e instructivo, aseguran la participación civil en la programación del presupuesto regional o local, en proyectos de alcance social que incluyen a la salud; cabe resaltar que la información sobre el uso de este presupuesto es pública y está disponible en el portal electrónico del Ministerio de Economía y Finanzas ${ }^{(2)}$. El porcentaje de proyectos en salud, en los PP desde el 2010 y para todas las regiones, se muestra en la Tabla 1 de donde se desprenden observaciones que destacamos más adelante.

Llama la atención que en algunas regiones como Huancavelica, Huánuco, Cajamarca, Madre de Dios y Piura, no hayan aumentado el número de proyectos en temas de salud dentro del PP, aun cuando estas regiones son consideradas de alta vulnerabilidad ${ }^{(3)}$. Aun más preocupante es que tres de ellas (Madre de Dios, Piura y Huancavelica) hayan disminuido el número de proyectos año tras año.

En general, el bajo número de proyectos en salud es congruente con lo reportado sobre la relación inversa entre tasas de desnutrición y mortalidad infantil, con la inversión per cápita en salud a nivel regional también en nuestro país ${ }^{(4)}$.

El decreciente número de proyectos en salud en el PP puede ser explicado por varias razones reportadas previamente ${ }^{(1,5)}$. Entre ellas tenemos la falta de voluntad

\footnotetext{
1 Facultad de Medicina “Alberto Hurtado", Universidad Peruana Cayetano Heredia. Lima, Perú.

2 Sociedad Científica de Estudiantes de Medicina Cayetano Heredia (SOCEMCH). Lima, Perú.

3 Escuela de Postgrado "Víctor Alzamora Castro", Universidad Peruana Cayetano Heredia. Lima, Perú.

a Estudiante de Medicina, ${ }^{\text {b }}$ médico magíster en Salud Pública y Epidemiología.

Recibido: 06-09-12 Aprobado: 31-10-12
}

Citar como: Carrillo-Larco RM, Segura ER. Presupuesto participativo, ¿las regiones más vulnerables lo invierten en salud? Rev Peru Med Exp Salud Publica. 2012;29(4):583-4.
Tabla 1. Número de proyectos en salud en los PP de todas las regiones. Perú, 2010-2012.

\begin{tabular}{|c|c|c|c|}
\hline \multirow{2}{*}{$\begin{array}{l}\text { Gobierno } \\
\text { regional }\end{array}$} & \multicolumn{3}{|c|}{ Proyectos priorizados por PP } \\
\hline & $\begin{array}{c}\text { Línea de } \\
\text { acción: salud }\end{array}$ & $\begin{array}{l}\text { Total de } \\
\text { proyectos }\end{array}$ & $\%$ \\
\hline Callao & 52 & 102 & $\overline{51,0}$ \\
\hline Cajamarca & 10 & 26 & 38,5 \\
\hline Puno & 29 & 128 & 22,7 \\
\hline Apurímac & 139 & 723 & 19,2 \\
\hline Amazonas & 16 & 86 & 18,6 \\
\hline Piura & 58 & 313 & 18,5 \\
\hline Huánuco & 20 & 116 & 17,2 \\
\hline Loreto & 10 & 66 & 15,2 \\
\hline Ucayali & 12 & 84 & 14,3 \\
\hline Arequipa & 69 & 549 & 12,6 \\
\hline La Libertad & 41 & 333 & 12,3 \\
\hline San Martín & 19 & 161 & 11,8 \\
\hline Pasco & 7 & 61 & 11,5 \\
\hline Huancavelica & 142 & 1278 & 11,1 \\
\hline Cusco & 31 & 289 & 10,7 \\
\hline Lima & 23 & 220 & 10,5 \\
\hline Lambayeque & 30 & 306 & 9,8 \\
\hline Tumbes & 26 & 286 & 9,1 \\
\hline Ancash & 59 & 663 & 8,9 \\
\hline Junín & 9 & 105 & 8,6 \\
\hline Ica & 21 & 251 & 8,4 \\
\hline Madre de Dios & 79 & 1150 & 6,9 \\
\hline Moquegua & 8 & 128 & 6,3 \\
\hline Tacna & 6 & 95 & 6,3 \\
\hline Ayacucho & 7 & 125 & 5,6 \\
\hline
\end{tabular}

Fuente: Dirección General de Presupuesto Público.

política de las autoridades que dirigen, o impulsan el PP; una sociedad civil poco participativa, o que se siente menos frente a su contraparte institucional. Respecto a lo último, el fomento de la participación ciudadana, y la coordinación con las autoridades locales en el desarrollo de políticas sociales, es parte de las funciones del primer nivel de atención (PNA), según la NTS 021-MINSA/DGSP-V.02. También, el PNA debe identificar las carencias en salud en su jurisdicción, elaborar planes y análisis, y socializarlos con la población. Así, puede buscar motivar en la población el ánimo por asumir una participación activa en el contexto de los programas sociales, regionales o nacionales, como lo es el PP.

La participación ciudadana, a partir del empoderamiento e información, promueve que la sociedad civil, muchas veces marginada, se beneficie del desarrollo social y económico ${ }^{(5)}$. En la actualidad las políticas, y proyectos para combatir los problemas de la salud pública no deberían basarse solo en teoría epidemiológica, cifras estadísticas, u oportunidades políticas; deben contar también con la participación directa de todos aquellos actores involucrados, incluyendo la sociedad civil. Los autores consideramos importante reforzar la actividad del PNA y esfuerzos similares en cuanto a informar y empoderar a la población 
para que busquen continuar, o aumentar, las iniciativas en salud en el PP. Lo mismo también es aplicable a otros programas sociales, en especial en aquellas regiones donde la situación de salud no tiene buenos indicadores.

Fuentes de financiamiento: autofinanciado.

Conflicto de interés: los autores declaran no tener conflictos de interés.

\section{REFERENCIAS BIBLIOGRÁFICAS}

1. Montecinos E. Democracia participativa y presupuesto participativo en Chile: ¿complemento o subordinación a las instituciones representativas locales? Rev cienc polít (Santiago). 2011;31(1):63-89.

2. Perú, Ministerio de Economía y Finanzas. Presupuesto Participativo [Internet]. Lima, Perú: MEF; [citado el 13 de agosto de 2012]. Disponible en: http://presupuesto-participativo.mef. gob.pe/app_pp/db_distedit.php

3. Perú, Ministerio de Salud, Dirección General de Epidemiología. Análisis de la situación de salud del Perú. Lima: 2010.

4. Monje-Vargas JA. Equidad e inversión en salud pública de la República del Perú. Rev Cubana de Salud Pública. 2011;37(4):452-61.

5. Parkinson S. Power and perceptions in participatory monitoring and evaluation. Eval Program Plann. 2009;32(3):229-37.

Correspondencia: Rodrigo M. Carrillo Larco.

Dirección: Av. 28 de Julio 818, Lima 18, Perú.

Teléfono: (51) 997951551

Correo electrónico: rodrigo.carrillo@upch.pe

\section{REACTIVIDAD CONTRA Treponema pallidum EN DONANTES DE SANGRE, IBAGUÉ, COLOMBIA, 2011}

\section{REACTIVITY TO Treponema pallidum IN BLOOD DONORS, IBAGUÉ, COLOMBIA, 2011}

\section{Harold Cruz-Bermúdez ${ }^{1, a}$, Sandra Forero-Rincon ${ }^{1, b}$, Jorge Moreno-Collazos ${ }^{2, c}$}

El uso seguro de los componentes sanguíneos es un constante reto para los entes reguladores de salud pública y, en general, para los bancos de sangre. En

\footnotetext{
Departamento de Investigación. Fundación Hematológica Colombia. Bogotá, Colombia.

2 Facultad de Enfermería y Rehabilitación, Universidad de la Sabana. Bogotá, Colombia.

a Enfermero, especialista en estadística aplicada; ${ }^{\text {b }}$ bacterióloga y laboratorista clínica; ${ }^{\mathrm{c}}$ magister en ciencias.

Recibido: 15-10-12 Aprobado: 31-10-12
}

Citar como: Cruz-Bermúdez H, Forero-Rincon S, Moreno-Collazos J. Reactividad contra Treponema pallidum en donantes de sangre, Ibagué, Colombia, 2011. Rev Peru Med Exp Salud Publica. 2012;29(4):584-5.
Colombia se encuentra estipulado en el Decreto 1571 de 1993, "Manual de normas técnicas, administrativas y de procedimientos en banco de sangre", la realización obligatoria de pruebas de tamizaje de las unidades de sangre, para detección de agentes potencialmente transmisibles por transfusión, como son: los anticuerpos para virus de la inmunodeficiencia humana $(\mathrm{VIH}) 1$ y 2; anticuerpos para hepatitis $\mathrm{C}$; antígeno de superficie para hepatitis B (HbAgS); serología para sífilis, y por último, anticuerpos para el Tripanozoma cruzi ${ }^{(1)}$.

La prevalencia de sífilis es variable, en países desarrollados resultados positivos a VDRL ocurren entre el 0,05 al $0,6 \%{ }^{(2)}$; en países africanos alcanza el $13,8 \%$; en Asia la proporción es de 5,8\%, mientras que en Latinoamérica alcanza valores entre 0,7 al 4,1\% ${ }^{(3-4)}$. En la mayoría de los casos los bancos de sangre y sistemas de vigilancia epidemiológica de estos países calculan la prevalencia según los casos sobre el total de unidades procesadas por los hemocentros, este cálculo se hace considerando la posibilidad de detectarse picos epidemiológicos en donde la infección se puede presentar con mayor frecuencia.

Con el objetivo de caracterizar a los donantes de sangre que presentaron reactividad contra Treponema pallidum durante el 2011 en un banco de sangre de la ciudad de Ibague, Colombia, se realizó un estudio transversal en el Banco de Sangre Fundación Hematológica Colombia, sede Ibagué. Se analizó el total de la población de registros de donantes de sangre que presentaron reactividad en el el tamizaje para Treponema pallidum durante el año 2011. Las variables analizadas fueron: edad, sexo, estado civil, régimen de filiación, estado civil, y ocupación.

En el análisis estadístico descriptivo se aplicaron medidas de tendencia central y de dispersión para las variables cuantitativas, y frecuencias absolutas y relativas para las variables cualitativas; se calculó la prevalencia de reactividad para $T$. pallidum. En el análisis bivariado se aplicó la prueba chi cuadrado. El desarrollo de la investigación cuenta con el aval de la institución y del comité de ética e investigación de la Fundación Hematológica Colombia; la investigación se enmarca en la categoría de investigación sin riesgo puesto que fue un estudio retrospectivo según la resolución 008430 de 1993 del Ministerio de salud de Colombia.

Durante el 2011 en la sede de Ibagué se procesaron un total de 37998 unidades de sangre en el Banco de Sangre Fundación Hematológica Colombia; se encontró una prevalencia de infección de 1,92\%, es decir 733 registros de donantes de sangre, de los 